\title{
NOTICE RE ANNUAL MEETING
}

On an exceptional basis, the 1973 Annual Meeting of the Canadian Political Science Association will not be held in June along with the other Learned Societies. Instead, it will be held at Sir George Williams University in Montreal on 18 and 19 August, the two days preceding the World Congress of the International Political Science Association, which runs 20-25 August. In addition, this year's annual meeting will be sponsored jointly with the Société canadienne de science politique and the program will consist of French, English, and bilingual sessions. It is our current expectation that the usual grant made to us by the Canada Council to support travel to the Learned Societies meetings will be transferred to the August meeting. In addition, we have requested a specific grant this year to help defray some of the extra costs associated with the long stay in Montreal for the World Congress.

These funds are particularly designed to help younger members on the assumption that those presenting, discussing, or chairing papers will be subsidized by their own university. Those requesting CPSA/ACSP grants are urged to obtain finance from their own institutions in order to allow the Travel Fund to be spread widely. Requests, stating the cost of the economy return air fare, the grants you expect to receive from other sources, and the academic rank of the member must reach the Secretary-Treasurer, Professor John Trent, Canadian Political Science Association, c/o University of Ottawa, 30 Stewart Street, Ottawa, Ontario, k1N 6N5, by May 30th, 1973. Please enclose a self-addressed, stamped envelope with your request.

\section{AVIS CONCERNANT LA REUNION ANNUELLE}

Exceptionnellement cette année la réunion annuelle de l'Association canadienne de science politique ne se déroulera pas en juin, comme celle des autres Sociétés Savantes. Elle aura plutôt lieu à l'Université Sir George Williams de Montréal les 18 et 19 août, c'est-à-dire durant les deux jours qui précèdent immédiatement le Congrès mondial de l'association internationale de science politique, qui se tiendra du 20 au 25 août. De plus, la réunion de cette année sera patronée conjointement par la Société canadienne de science politique et le programme comprendra des séances en langue française, en langue anglaise, et des séances bilingues. Nous espérons que la subvention que nous accorde habituellement le Conseil des Arts afin de défrayer le coût des voyages pour permettre aux gens d'assister aux réunions des Sociétés Savantes sera reportée pour notre réunion en août. Nous avons en outre demandé au Conseil une subvention majorée cette année afin de nous aider à rencontrer les coûts plus élevés entrainés par un séjour prolongé à Montréal à l'occasion du Congrès Mondial.

Ces fonds sont d'abord mis à la disposition des membres plus jeunes, attendu que le voyage de ceux qui présentent ou discutent une communication, ou qui y président sera vraisemblablement défrayé par leur propre université. Ceux qui désirent se prévaloir de cette aide devraient tâcher d'obtenir d'abord une subvention de leur employeur, ce qui permettrait à CPSA/ACSP de répartir son assistance plus largement. Les demandes de subvention devraient, après avoir précisé le coût du transport aérien (aller-retour, classe économique) et déclaré les autres sources de financement escomptées, ainsi que le statut académique du demandeur, être envoyées au secrétaire-trésorier, Professeur John Trent, Association canadienne de science politique, Université d'Ottawa, 30 rue Stewart, Ottawa, Ontario, K1N 6N5, pour le 30 mai 1973, accompagnées d'une enveloppe aflranchie. 